\title{
The Right Lateral Frontal Cortex Processes Features of Emotional Faces as a Function of Their Likelihood of Occurrence
}

\author{
André Forster*a, Johannes Rodrigues ${ }^{a}$, Philipp Ziebell ${ }^{a}$, Joseph L. Sanguinetti ${ }^{b}$, John JB. Allen ${ }^{b}$ and \\ Johannes Hewig ${ }^{a}$ \\ ${ }^{a}$ Julius Maximilians Univeristy of Würzburg, Marcusstraße 9-11, 97070 Würzburg, Germany \\ ${ }^{b}$ University of Arizona, Tucson, USA
}

\section{ARTICLE INFO}

Keywords:

riFG

transcranial Ultrasound

Emotion Processing

Alpha

\begin{abstract}
A B S T R A C T
The lateral frontal Cortex serves an important integrative function for converging information from a number of neural networks. It thus provides context and direction to both stimulus processing and accompanying responses. Especially in emotion related processing, the right hemisphere has often been described to serve a special role including a special sensitivity to stochastic learning and model building. In this study, the right inferior frontal gyrus (riFG) of 41 healthy participants was targeted via ultrasound neuromodulation to shed light on the involvement of this area in the representation of probabilistic context information and the processing of currently presented emotional faces. Analyses reveal that the riFG does not directly contribute to processing of currently depicted emotional stimuli but provides for information about the estimated likelihood of occurrence of stimulus features.
\end{abstract}

\section{Introduction}

The processing of emotional stimuli has been subject to extensive research in the translational neurosciences. One cortical area strongly involved in the general evaluation of (un)emotional stimuli and the individual's response to these is the lateral prefrontal cortex (IPFC, [22]). The IPFC is often recognized for its integrative capability, constructing a consistent model of the world from a variety of information and stimulating consistent and goal directed responses ([26]; [38]). It has (among other results) been reported to transmit information regarding mood ([70]), motivation ([43]; [29]), memory ([5]; [6]) and inhibitory control ([37]; [89]). Even though a large number of studies have been conducted to clarify its role in these and other fields of research, the IPFC's functional dissociation into specialized subregions and networks as well as its hemispheric specificity is still up to substantial debate (e.g. [22]; [34]; [51]; [51]). Furthermore, a unifying framework associating these subregions and their respective networks with complementary steps in the processing of emotion and emotional stimuli is still lacking. This may in part be owed to the complex patterns of network interactions that can lead to contradictory or nullresults as well as to differences in methodology used in the current literature ([72]).

One increasingly utilized way to partly disentangle such patterns is the neuromodulation of narrowly circumscribed areas. Therefore, the present study seeks to provide insights into the unique contribution of the right inferior gyrus of the IPFC (riFG) to the reaction to and processing of emotional stimuli by modulating its excitability via low intensity transcranial ultrasound neuromodulation (litFUS). The main focus of this investigation is the right hemisphere's supposedly unique capability to model stochastic information

\footnotetext{
*Corresponding author

@ andre.forster@uni-wuerzburg.de (A. Forster*) ORCID(s):
}

and experience, which may reflect processing of contextual information about currently presented emotional stimuli.

\subsection{Specialization of the Right IPFC}

Research on frontal asymmetry (FA) suggests a relative rather than an absolute interpretation of hemispheric activity. It mostly but not solely builds on an EEG index that is calculated by subtracting the natural logarithm of the alpha power in left hemispheric electrodes (usually F3 or F7) from right hemispheric ones (F4 or F8). Positive values of the resulting measure indicate greater left than right $1 \mathrm{PFC}$ activity, while negative scores imply greater right than left cortical excitation. Furthermore, even though the theory behind FA has notably evolved throughout the past decades ([35]; [89]; [18]; [37]) an overall consensus on its correlation to motivational states has been established ([43]; [73]). As approach and withdrawal motivation go along with certain emotions, a substantial body of literature further describes its implications in mood and emotion processing ([70];). Moreover, FA has been researched in a number of contexts including a variety of disorders like depression ([2]), ADHD ([45]) or anxiety ([80]) hinting to its role as a possibly traitlike vulnerability to disorders that comprise some sort of dysregulation of motivational tendencies ([86]; [82]; [64]; [39]; [36]) but also see: [88]).

Other studies focused on one hemisphere at a time instead of their relative activity. On that note, for the right IPFC two hypotheses emerged, one stating a general preference of the right hemisphere for emotion processing ([11]; [21]; [32]; [81]; [90]; [97]), while the other suggests a specific involvement in negative or generally withdrawalrelated emotions ([91]; [96]; [4]; [69])

In addition, Yang et al. ([97]) point out that both hypotheses may coexist in the way that a special sensitivity for negative emotional processing is build on top of the general processing capability of emotions inside of the right IPFC. In conclusion, even though the discussion on the correct 
mapping of emotional processing to either side of the IPFC is still ongoing, a consensus emerges that the right IPFC plays a crucial role at least in the processing of negative emotion and negatively valenced stimuli.

Moreover, the (bilateral) IPFC shows another specialization, which is attention modulation (e.g. [66]; [40]; [13]). In general, IPFC dependent attention processes show mood and context congruent selectivity, which is highlighted by reports on differences in attention modulation in patients suffering from depression, PTSD, addiction and other diseases (e.g. [23]; [25]; [98]). A few studies using neuromodulation techniques show a specialized right hemispheric involvement in the detachment from emotional faces in general with a greater effect for angry faces. More precisely, these results describe greater right $\mathrm{PFC}$ activity to impair attentional shifting away from emotional stimuli. ([56]; [67]; [76]).

Finally, a number of studies described the specificity of the right IPFC for probabilistic learning ([27]) as well as stochastic model building and updating ([26]) including evidence for impairments in stochastic model building and updating in brain damaged and split brain patients ([94]; [61]; [74]; [17]). In addition, [83] report no such impairment in patients with left sided brain damage.

In summary, the IPFC has been described to play an important role in the processing of emotions, motivation and attention with a hemispheric specification for non-verbal stochastic representations of the recent history of events for right lateralized networks including right prefrontal areas. Taken together, these insights highlight the bilateral IPFC's and specifically the right IPFC's importance in psychopathological states as the prolonged or excessive display of fear, dysphoria, withdrawal and many other symptoms may be a result of a highly entangled combination of these features. For instance, an impaired representation of the probability for positive reinforcement due to an excessive activity in the right IPFC, may lead to decreased approach motivation, increased anxiety or sorrowfulness. As a result, a mood congruency effect facilitating attention focusing on and learning from negative events may follow. However, to this date, no process model detailing such sequences of processing steps has been described. For this reason, this direction of action could also occur in reverse: A mood effect may contribute to the display of congruent behavior (withdrawal, worrying, etc.), which could ultimately lead to an altered representation of matching emotional stimuli. In summary, all of these consequences may by themselves be the starting point for such sequential or concurrent stages of processing, leading to the symptoms described above.

\subsection{Emotional Faces and Depression}

One set of stimuli combining many features that are supposedly linked to right IPFC processing are faces depicting emotional states ([76]; [75];). [97]). Not only does it provide ground for emotional, non-verbal learning, which plays a crucial role in the pathogenesis of depression, a highly social disorder, but it may also disentangle emotional processing from those of other features, like sex (which may arguably also include emotional associations, see section 4).

Depression describes a very heterogeneous state that goes along with increased processing of negative stimuli and impaired social functioning ([95]). As a result, over the last decades, several studies have focused on depressed individuals to investigate neural substrates of diverging processing of both emotional and social cues (e.g. [19]; [57]; [47]; [48]). As the frontal asymmetry of IPFC activity and activation has previously been described to play a role in trait-vulnerability for depressive episodes, motivational states and affect ([70]), results stemming from the literature on the $\mathrm{PFC}$ conveying emotional face processing and general neural substrates of emotion and motivation may converge at this area. One example is the study by Kerestes et al. ([44]), who described the time elapsed after the last depressive episodes to be associated with decreased left-sided IPFC activity in response to anxious compared to neutral looking faces. This fits the idea of FA research, which shows that depression is associated with a less left-compared to right sided IPFC activity ([36]). Although this difference may be due to several reasons, for example less left frontal activity or more right frontal activation, these correlative findings in studies comprising individuals showing trait vulnerability to the over-representation of negative affective cues when viewing emotional faces are in line with experimental evidence. For instance, [76] report decreased gaze disengagement from negative cues in emotional faces following tDCS conveyed excitation of the right dorsolateral prefrontal cortex.

Another study addressing the relationship of negative affect in emotional faces and neural responses is provided by [69]. The authors conducted two experiments that manipulated both, predictability of the emergence of fearful or happy faces as well as the participant's attention to these. Their results indicate a right- hemispheric attention- modulated neural response to fearful faces and a left-hemispheric sensitivity to happy faces.

Accordingly, the current study also used a set of emotional faces as stimuli. They were presented in a protocol described by Lissnyder et al. ([58]), who examined sequence effects of emotion expression in comparison to sex in context of cognitive shifting capabilities. In summary, in blocks of 11 faces, a random sequence of stimuli was presented. These depicted either a male or female, angry or neutral looking face. The task consisted of participants trying to internally count either the number of angry/neutral looking faces or male vs. female faces as fast as they could (the focus of counting altered for each experimental block). Thus, in a given block, explicit processing of one feature (i.e. sex), but not the other (i.e. emotion) was primed.

\subsection{Low Intensity Transcranial Ultrasound Neuromodulation}

litFUS is a novel method to alter neural excitability. Unlike other methods such as TMS or tDCS, litFUS uses mechanical waves (sound) to oscillate neural tissue and thereby influence its reactivity. Even though to this point 
no conclusive mechanism of effect has been established, several ideas about mediating biophysical properties of neurons have been formulated. For instance, mechanosensitive receptors like the two-pore-domain-potassium channels of the TREK family were shown to react to the ultrasound induced conformatory changes in cell membranes ([50]). Furthermore, several other cellular structures have been hypothesized to convey the effect due to their mechanosensitivity ([87]).

The model used to estimate the litFUS effect in this study is described by the NICE-model, which predicts inhibitory effects to result from the excitation of inhibitory interneurons that express a large number of T-type calcium channels, thus leading to a net inhibition from a network level point of view([65]). Interestingly, these channels have also been described to influence the general EEG alpha response and account for individual differences in the individual alpha peak frequency (IAF) as T-type channels fire at a rate of approximately $10 \mathrm{~Hz}$ (see [8]). Therefore, by exciting these channels, increases in the alpha-band (which is inversely correlated to cortical activity) power may emerge following inhibitory litFUS modulation. Throughout the past years a few but steadily increasing number of studies used this method to safely modulate neural tissue in humans (in vivo, [33]; [54]; [53]; [55]; [77]; [71]; [52]).

\subsection{The current study}

Building on the aforementioned remarks, this study examines the unique properties of the right IPFC regarding probabilistic processing of non-verbal information and its modulating role in emotion processing. Accordingly, the present study used emotional faces to elicit stimulus-driven EEG oscillations on a single trial level, which allowed for the investigation of the influence of stimulus probability on the reaction to a given trial.

Thus, we hypothesized EEG alpha oscillations, which are part of the frequency range that is targeted by our settings of litFUS, to be modulated by the likelihood of stimuli. Furthermore, since many studies reported the right IPFC to be especially involved in emotion processing, this effect should also be present in the probabilistic processing of emotional features. Nonetheless, as many studies investigating the stochastic learning capability of the right hemisphere also reported positive results for stimuli and tasks without emotional features, we also assume this effect to be present regarding the sex of depicted faces. In conclusion, the alpha response at F8 should depend on an interaction of the estimated likelihood of occurrence regarding a specific emotion and a certain sex. Furthermore, the alpha response to this interaction should also be altered depending on the focus of attention (on either sex or emotion). Hence a three-way interaction is anticipated between the likelihood of occurrence of sex, emotion and attention focus. Moreover, this interaction (targeting the current history of experiences) may additionally impact the processing of the currently depicted emotional faces, leading to a four-way interaction. At last, this already complex term may be modulated as a whole or in part by the ultrasound neuromodulation, which would further strengthen the idea of the direct involvement of the right IPFC (more specifically the right inferior frontal gyrus [riFG]) in these processes.

As a result our hypotheses (concerning the alpha power density at F8 electrode position as dependent variable) are modeled including a four- way interaction of litFUS, likelihood of an emotion and sex of a depicted face and focus of attention, as well as another four-way interaction comprising litFUS, the currently depicted emotion, focus of attention and the estimated likelihood of an emotion. This split into two separate interaction terms was chosen as the currently depicted emotion specifically is not thought to rely on the estimated likelihood of a given sex to be depicted. Thus in order to reduce the already complex pattern of interactions, a five-way interaction was avoided leading to the present solution.

In addition, since inactivity of the riFG is in general interpreted to signal decreased emotion processing (depending on the the theory, either for emotions in general or specific to withdrawal-related ones) we also hypothesize the right IPFC's alpha response to be inversely correlated to reaction time (RT). Furthermore, even though no directed idea is formulated for the influence of litFUS, an interaction of the neuromodulation and alpha is anticipated for RTs. In addition, litFUS is thought to modulate RT differences between sequences of faces. On that note, decreases in RT in trials comprising shifts from angry to neutral faces in comparison to other sequences were found to correlate to trait worrying, which may build on impaired capabilities to detach from the negative/threatening face of the previous trial ([58]). As a result, since litFUS is thought to decrease the processing of (negative) emotions, we anticipate an interaction of litFUS and sequence of depicted emotions. At last, this effect may vary depending on the focus of attention.

\section{Methods}

\subsection{Sample.}

Participants were recruited from a stock of test-persons registered to a website specifically set for the purpose of enrollment in psychological studies hosted by the University of Würzburg, Germany. Forty-one right-handed, healthy participants, who were at least 18 years of age took part in this study ( 29 female) including 36 undergraduate or graduate students (23 students of psychology). Participants received either course credit or $15 €$ as a compensation for taking part in this study. Average age was $24.46(\mathrm{SD}=8.58)$. Mean BDI-V ${ }^{1}$ scores were 22.8 , mean IAF was 10.0 The procedure of neuromodulation was assessed by the psychological institute's ethical committee and deemed ethically unproblematic (reference: GZEK 2017-18). Informed consent (also including publication of their respective data) was given by all participants before taking part in the experiment.

\footnotetext{
${ }^{1}$ the BDI-V is a questionnaire building on the BDI-II. It is especially suited for subclinical differentiation. Individuals with scores over 35 are considered to show a clinically relevant depression with a sensitivity of 90\%;[78];[79].
} 


\subsection{Material.}

For the Internal Shift Task (IST) 24 image files from the KDEF collection were used (KDEF; [59]). The image files contained 19 aggressive looking faces (9 male and 10 female), and 20 image files with neutral expressions of faces (9 male and 11 female).

\subsection{Procedure.}

Participants completed an online survey before visiting the laboratory in order to give them the opportunity to finish questionnaires in a natural setting, thereby preventing possible biases that arise from interactions of the lab-setting with personality variables (e.g. inducing worrying in testpersons who might become unsettled by the general labsetting). With the exception of the BDI-V ([78]), none of the questionnaires were used for this study. However, the present experiment was part of a battery of three independent tasks that did not change in their sequence of presentation between participants or between sessions. The current task was always presented as the second of three. No stimulus material of the task was reused in other tests. No other task comprised faces or angry looking nor anger eliciting stimuli.

After completing the survey, participants attended two experimental sessions (exactly seven days apart) consisting of the same task and procedure. However, on one measurement occasion, participants received litFUS modulation while sham was applied in the remaining one. The experiment thus represents a double blind cross over design with two groups that differ in their respective session of modulation ( $\mathrm{t} 1$ or $\mathrm{t} 2$ ). Unfortunately no exact measure of the time passed between administration of litFUS to the start of the task can be provided. However, approximately 30 to 45 minutes are estimated based on the time it took to prepare the passive EEG electrode setup (which introduces the most variability to the estimate) and to complete the preceding task (less than 10 minutes). Following litFUS or shammodulation, EEG electrodes were administered. Participants were seated in a distance of approximately $60 \mathrm{~cm}$ from a $24-$ inch screen. Subsequently, testing of participants began.

Internal shift task. The internal shift task (IST, [58]) was one of three independent tasks in a larger assessment of effects of litFUS-modulation. During the task, participants are instructed to (internally) either count the number of angry and neutral faces or to count the number of female and male faces presented ([58]).

Displayed faced (326 x 326 pixels) were randomly chosen (with replacement) from a subset of 19 neutral and 20 angry faces selected from the Karolinska Database of Emotional Faces ([59]). This subset equaled the selection of the 20 most valid pictures per emotion as depicted by [30] with one exception (AF16 in neutral condition). Whether sex or emotion is in the focus of internal counting during one block alternated and was indicated before the next one started. Moreover, in order to rule out effects from contextual cues, hair, clothing and background were cropped out of the pictures.
A total of 24 blocks, each consisting of 11 trials was completed in one session with half of the participants starting with sex and half starting with emotion in focus. To correct for sequence effects, participants were randomized to either group and began in different order of blocks on session two.

In each trial, after they are finished updating the two numbers they were supposed to keep in mind, participants pressed space to continue to the next picture so they can update their numbers in mind again. After pressing space, a fixation cross was presented for $200 \mathrm{~ms}$ before the next face appeared. Following each block, participants were asked how many angry vs. neutral faces or male vs. female faces they had counted. Subsequently, test subjects were told what was to be counted during the next block. The procedure is illustrated by Figure 1

\subsection{Transcranial Ultrasound Neuromodoulation.}

Neuromodulation was applied at F8 electrode position of the 10-20 EEG system. This location was chosen not only because of the underlying riPFC an area with strong implications in the cognitive control network ([15]) and frontal asymmetry but also due to the underlying temporal window of the skull. This structure is (compared to other skull locations) relatively thin making it less likely to distort or absorb energy.

Neuromodulation was applied for 120 seconds with a duty cycle of $0.5 \%$, a frequency of $500 \mathrm{kHz}$ and a pulse repetition frequency of $40 \mathrm{~Hz}$ leading to an acoustic intensity spatial peak time average of $199 \mathrm{~mW} / \mathrm{cm}^{2}$ and a mechanical index of 1.53. The ultrasound was emitted by a single element transducer (with a two-part lens focused at $30 \mathrm{~mm}$ ) that was connected to a manufactured gel pad (product number SS-6060 by Silicone Solutions, Cuyahoga Falls, Ohio, USA), which was in turn directly placed on the F8 electrode position of the scalp. Neuromodulation was operated by a manufactured device (Thync, Los Gatos, USA) and carried out by two experimenters at a time (one fixating the transducer and the gel pad to the participants head and one starting the procedure on the device). While the two minutes of modulation/ sham were running, participants were asked not to move or talk in order to prevent distortions of the transducer relative to the targeted location. The same device has been used before by Sanguinetti et al. ([77]). Further detail on its utilization and profile of emission may be reviewed there.

Since ultrasonic waves are too high in frequency to be heard by humans, litFUS can easily be shamed. In this study, experimenters either pointed the transducer at the desired region of interest or in the opposite direction, away from the participant's head. However, litFUS was emitted either way, keeping the preparation process for experimenters constant across litFUS- and sham-modulation. Nonetheless, the experimenters were not aware of which direction would direct energy from the transducer, thus allowing for a doubleblinded protocol. 


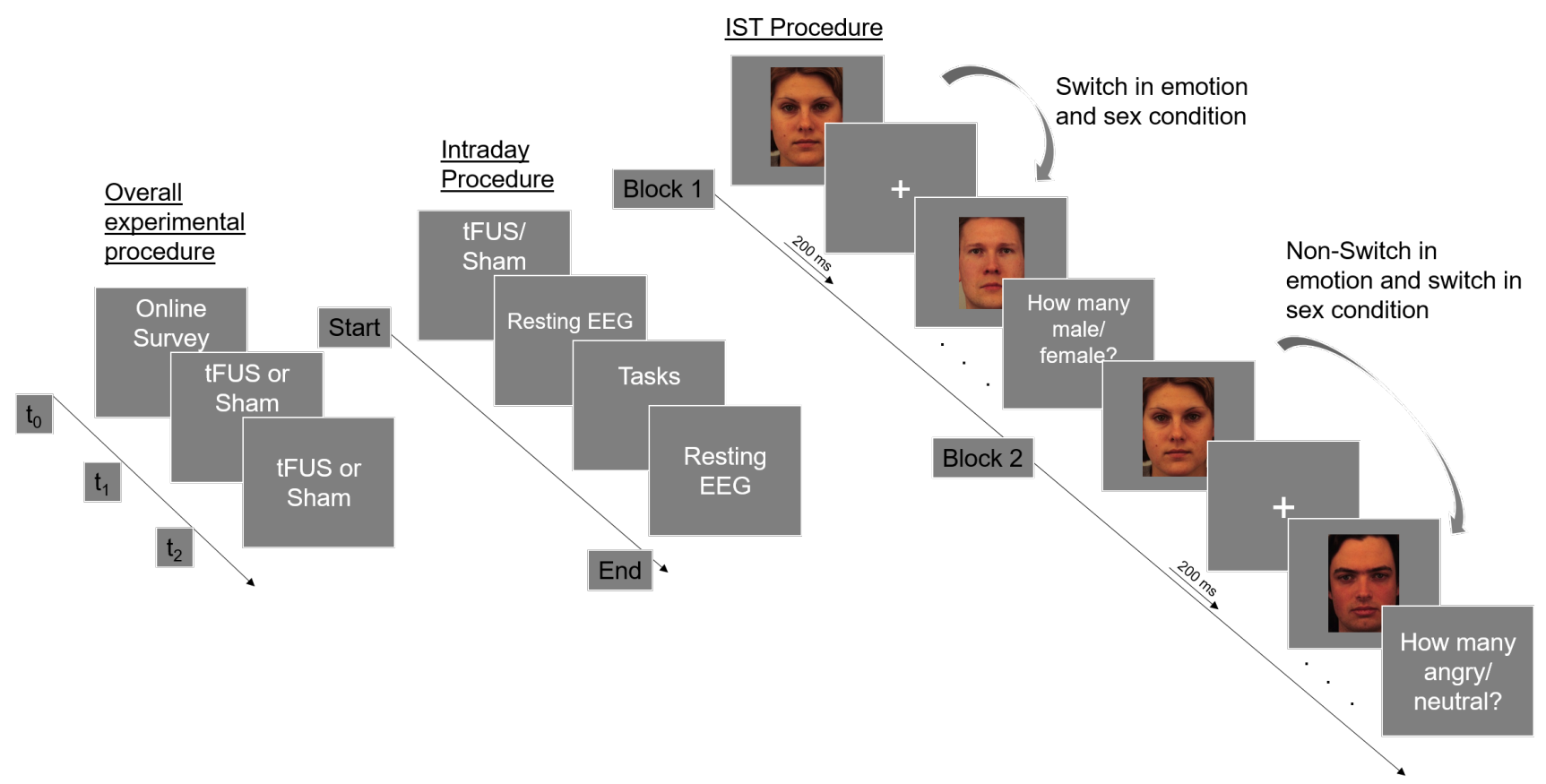

Figure 1: Conceptual representation of the overall-, intra-day- and task procedure. This experiment was the second of a total of three tasks within a test-battery. The same test-battery was presented two times, once under the influence of litFUS, once following sham. THe stimuli of this study were not used in other parts of the test-battery. Each session started and ended with a resting EEG of 8 minutes, which is beyond the scope of this paper. The present task comprised 24 blocks, each consisting 11 presented faces with an alternating focus on either sex or depicted emotion of shown faces. In this figure, two trials are presented within 2 blocks that differ in their focus on sex or emotion. The dots depicted before the question for the number of internally counted features indicate that he sequence of image depiction and fixation cross presentation continued 11 times.

\subsection{EEG administration and preprocessing.}

EEG recordings were collected via a 64 electrode brain cap system and recorded via the brain vision recorder (Brain Products, Gilching, Germany). However, due to technical issues, 13 participants were tested with an 32 electrode cap but an otherwise equal recording system. Nonetheless, according to Bazanova and Vernon ([9]), the cap montage does not influence alpha-related measures. Also, all following analyses rely solely on those electrodes that were included in both sets. Electrodes were brought under $5 \mathrm{k} \Omega$. Online reference was $\mathrm{Cz}$, ground was Afz. An online $80 \mathrm{~Hz}$ low-pass filter was applied. The sampling rate was $250 \mathrm{~Hz}$.

The preprocessing pipeline was executed via the Matlab (The MathWorks, Massachusetts, USA) extension EEGLAB ([20]) including plugins MARA ([93]), Adjust ([62]), SASICA ([12]), the CSD-toolbox ([41], [42]) and the restingIAF toolbox ([16]). The procedure followed the EPOS preprocessing pipeline of Rodrigues et al. ([72]): As a first step, electrodes were re-referenced to average. Following this, channels were automatically rejected and interpolated depending on their averaged z-score on the three dimensions kurtosis, probability and spectrum. A z-score (calculated for the mean of one channel in comparison to all others) of more/less than \pm 3.29 (following suggestions by [84] on outlier detection) qualified a channel for rejection. Epochs were extracted (-300 ms to $1200 \mathrm{~ms}$ ) and a $1 \mathrm{~Hz}$ high-pass filter was applied. Then, an ICA was conducted, and trials were automatically rejected by the same procedure as the channels had been. Following this, ICA weights and indices of rejected trials were saved. The preprocessing started once again and applied the saved parameters to the new set that was now missing the $1 \mathrm{~Hz}$ filter in its pipeline. Afterwards, SASICA was used to reject components based on MARA and Adjust evaluations of the data. At last, the data was CSD-transformed.

Following this, Morlet wavelets (fixed cycles of 3.5s, log-spaced) were used to extract the power of frequency bands, that were identified individually for each participant by analyzing an eyes closed resting condition that preceded the experimental test battery at each session via the restingIAF toolbox ([16]). IAFs and band width were determined by averaging calculated IAFs across a minimum of 17 channels (15 in 32 electrode setup) and a frequency range of 1-40 $\mathrm{Hz}$. Bounds of the IAF search window were set to $7-13 \mathrm{~Hz}$ and a Savitzky-Golay filter of 11 bins was administered. The IAF was then used to split the alpha band into two parts: upper alpha (IAF to upper band border) and lower alpha (low band border to IAF). Afterwards, the both measures were added up and the respective contribution of each band to this sum was calculated. At last, the general alpha response was expressed as a weighted composite of both bands multiplied with their respective contribution-parameter. All EEG measures were normalized via natural logarithms. All further analyses regarding EEG measures build upon the average between 100 and $300 \mathrm{~ms}$ after stimulus onset. 


\subsection{Measures.}

For the following analyses a number of measures are included that may not be obvious in their interpretations. Thus, this section provides an overview of the author's perspective on the reflected effects.

Stimulus presentation rates. This task presented a total of 264 faces in random order. Each face exhibited a combination of specific features which were summarized to the categories of emotional expression (angry vs. neutral) and sex (male vs. female). As a result, for each given trial a rate of the presence of a certain feature was calculated by the number of features present thus far divided by the number of trials thus far. The concluding moving average produces a number between 0 and 1 that in this study reflects the overall rate of male and angry faces. The these rates were calculated independently for the feature sex and emotion. Also, calculation of these measures did not consider whether a given trial urged particpants to process a feature explicitly or implicitly. We interpret these rates as the objective representation of the presentation of stimuli, which may provide data for subjective interferences about the likelihood of feature presentation in current or future trials.

Block. The experiment asks participants to either focus on the expressed emotion or the sex of presented faces. This focus of processing changes after 11 trials. As explicit processing of one feature and inhibition of processing of the other feature is part of the task, we conclude the block to represent a shift in selective attention. Explicit vs. implicit processing, block and shift of attention are thus used interchangeably throughout the text.

Emotion. The variable emotion describes the valence of the currently expressed emotion by the presented face in a given trial. It thus marks the immediate response to the currently depicted stimulus, neglecting sequence effects.

Trial. in the following analyses, Trial refers to the cumulative trial, given by the overall index of a given trial within one session. It rises continuously without interference of block or other variables and reaches a maximum of 264 (24x11).

Sequence. Sequence describes the sequence of depicted emotions from the current trial and the one before. As a result, four sequences emerge (angry $\rightarrow$ neutral, neutral $\rightarrow$ angry, neutral $\rightarrow$ neutral, angry $\rightarrow$ angry). Following the theoretical remarks of De Lissnyder, Koster and De Raedt, ([58]), especially the relation of angry $\rightarrow$ neutral to the other sequences should be correlated to worrying, depression and anxiety as participants scoring high on these constructs may have trouble shifting their attention away from the threatening stimulus, which may conclude in slowed reaction speed.

\subsection{Statistical analyses.}

In order to answer the questions raised by the hypotheses, two sets of mixed effects models with an increasing number of parameters were calculated. All models comprise a random intercept for each test-person, a random slope for trial and grand mean centered metric predictor variables. The nominally scaled variables Emotion, litFUS and Block were dummy coded to the reference levels of neutral, sham and focus on emotion/explicit processing of emotion, respectively. Thus, main effects as well as interactions of third variables not including one of these variables, can be interpreted in the fashion that the given effect is present in the given reference category. For instance, a significant main effect for emotion reflects a difference between neutral and angry faces in the sham condition. However, in order to test for the same effect in litFUS trials, the interaction term comprising both, emotion and litFUS needs to be consulted. The sequence of faces was simple-coded, introducing the grand mean of all levels as intercept (as opposed to dummy coding, which sets the mean of the reference level as intercept). Hence, other effects may be interpreted in light of no particular sequence of faces. Variables coding feature presentation rates, such as the rate of male or angry faces (Tables2 and 6) represent of the last trial before the current one (trial -1). Hence, the variance of the current trial, which is reflected by the variable emotion would not be included by the rate-measures. As a result, feature rates give more of a context to the given trial, while emotion catches the immediate response to the current event selectively.

Following the data-driven approach of mixed effects modeling, only the best fitting model was analyzed for fixed effects estimates. All models started with a random intercept (for each participant) and a random slope (trial within session).

Resulting from the theoretical foundation introduced above, a complex pattern of interactions may be present including a four-way interaction of the rate of angry faces, rate of male faces, block and litFUS. However, also lower order interactions of this term may emerge as well as main effects. Including all of these into the model may lead to substantial multicollinearity. Nonetheless, as this issues will generally decrease the likelihood of effect detection rather than inflating its probability, this way is estimated to be more conservative for a first investigation on this topic. All (nonexploratory) effects were Bonferroni-Holm adjusted with the exception of the estimate for the model's intercept, since no hypotheses were calculated for these. Furthermore, additional exploratory analyses were conducted to further clarify confirmatory results. Only those trials, that were within 3.29 $\mathrm{SD}$ from the grand mean of reaction times were included in the following analyses in order to exclude those that comprised some sort of distraction (leading to excessively high reaction times) or improper stimulus processing (by pressing before cognitive processes could take place).

All analyses were conducted via $R$ 's (R Core Team, 2017) lme4 ([7]) package and plotted with the effects([28]) package.

\section{Results}

Before reporting the results of above stated hypotheses, topographical plots for all conditions of this experiment are shown in Figure 2. This figure illustrates that the topographical map of alpha responses was in general more dependent 
Processing of Emotional Features in the Lateral Frontal Cortex

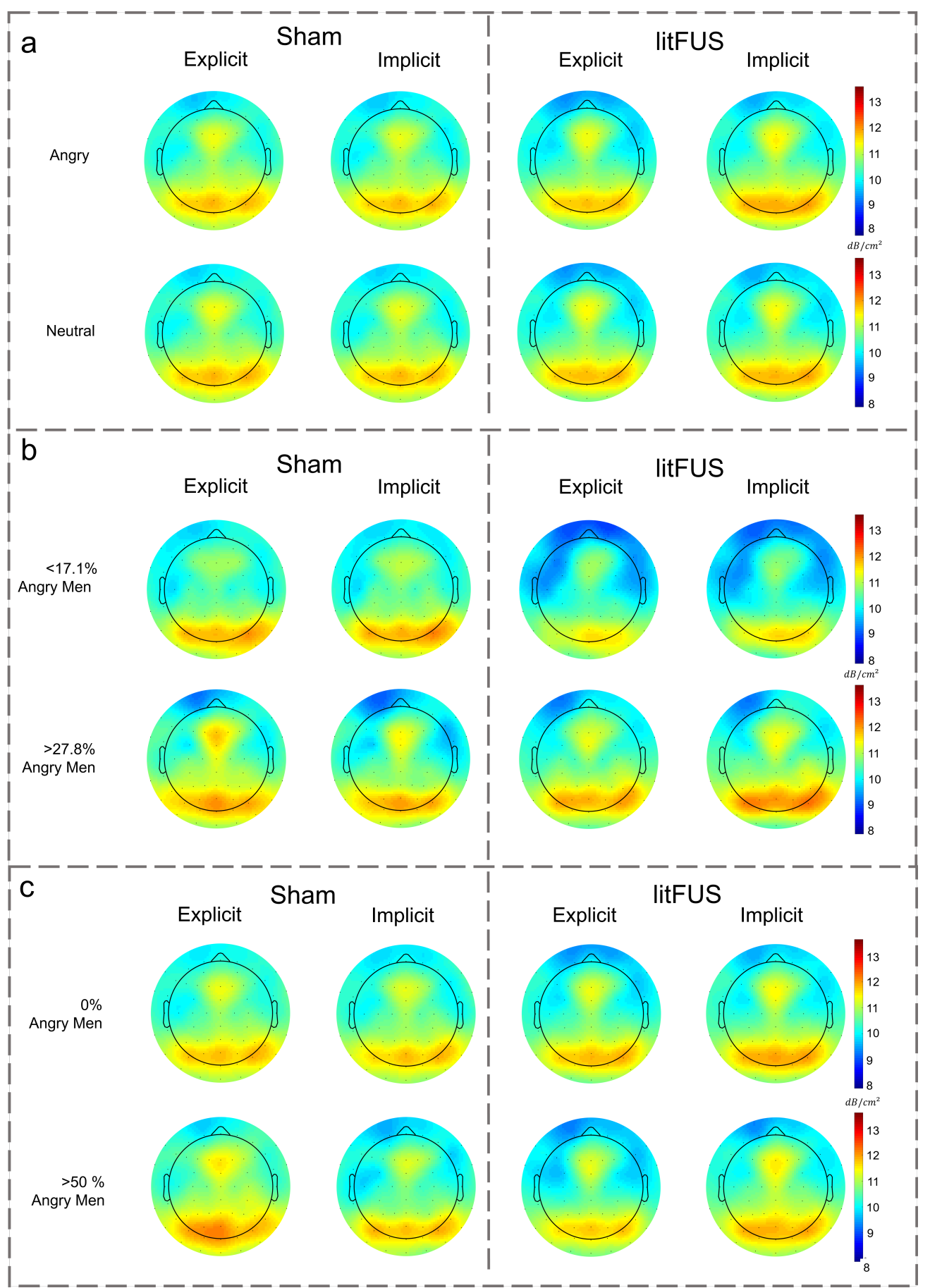

Figure 2: Topographical plots of the alpha response to experimental conditions. Colors represent $\mathrm{db} / \mathrm{cm}^{2}$ averaged from 100 to $300 \mathrm{~ms}$ after stimulus onset. a) Alpha activity following explicit or implicit presentation of angry or neutral faces. Only small fluctuations of activity is present within the sham and litfus conditions. However, less alpha activity seems present in both frontopolar and right IPFC regions when comparing the overall litfus and sham activity. Overall, the topographic response in the EEG seems to be hardly dependent on the currently presented emotion. b) Alpha activity in trials in which no more than $17.1 \%$ of the previous trials (representing the lowest $10 \%$ of rates found in the complete data set) or at least $27.8 \%$ percent (representing the highest $10 \%$ of rates found in the complete data set) of all images shown thus far depicted angry men. Plots (comparing high vs. low. rates among explicit and implicit blocks) indicate decreases in alpha activity at right prefrontal areas if more angry men have been shown before. This is true for both implicit and explicit feature processing but seems more pronounced in implicit emotion processing trials. c) Alpha activity in trials within one block where no angry men have been shown (representing the lowest $10 \%$ of rates) or at least $50 \%$ percent (representing the highest $10 \%$ of rates found) of images shown thus far within a given block depicted angry men. The pattern depicted in $b$ is also present in context of within-block likelihoods but seems less pronounced than in $b$, where the overall likelihood within one session instead of block was plotted. This indicates differences in right IPFC alpha activity in response to session-spanning likelihood of features assessments. 


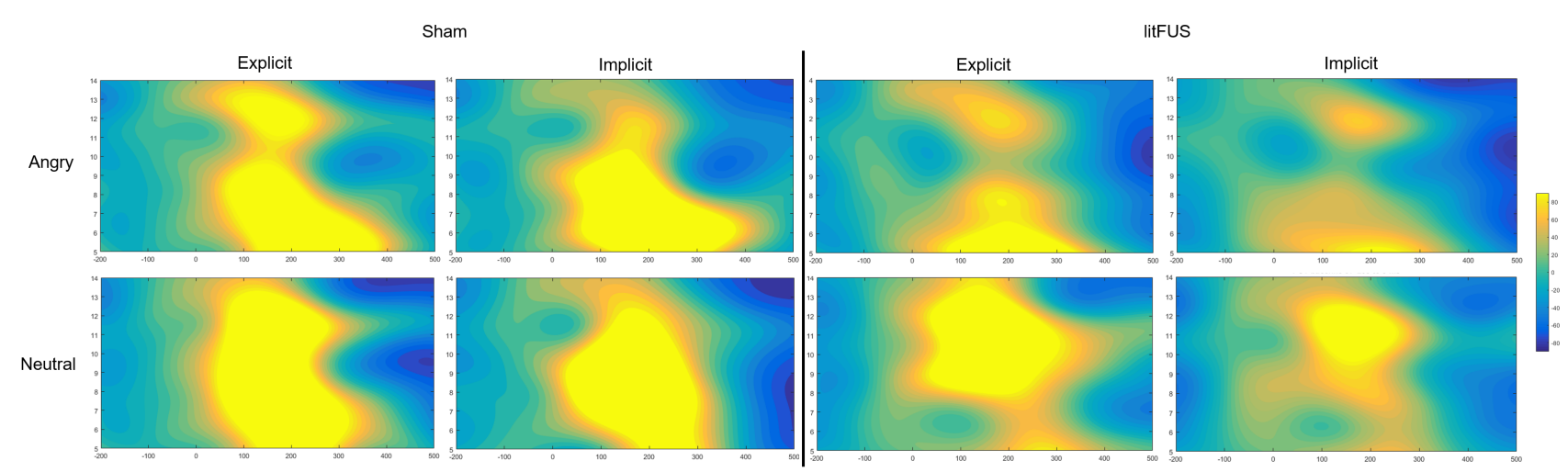

Figure 3: Time-frequency plots of experimental conditions at electrode position F8. Time-frequency plot averaged across implicit and explicit trials as well as across depicted emotions. The images show decreased alpha and theta responses to angry faces during litFUS sessions as compared to sham measurement occasions. Also, an alternated response in lower frequencies following neutral face depiction seems evident.

on the likelihood of occurrence of features than on the depicted face within the current trial: Regarding the alpha response to the currently depicted emotion, no prominent difference between plots is present within the sham and litFUS conditions. While litFUS seemed to decrease alpha at frontopolar and right IPFC regions, no other effect of emotion or implicit/explicit is evident (Figure 2a). Figure $2 \mathrm{~b}$ further illustrates decreasing alpha responses at the right IPFC in trials that followed a history of lower angry men rates as compared to those with higher rates during sham sessions (comparing the upper to the lower sham topoplots). However, this effect is reversed within litFUS sessions as the right $\mathrm{IPFC}$ increases its activity with higher rates of previously depicted angry men (comparing upper to lower topoplots within the litFUS condition). Figure $2 \mathrm{c}$ also shows, that the effect was similarly present when comparing blockwise rates of previously shown angry men. Nonetheless, the effect was most prominent when looking at the overall probability within the complete session rather than feature likelihoods within one block.

In line with these plots, Figure 3 illustrates the time frequency plots for each experimental condition at electrode position F8. These indicate a possible effect at at frequencies in the alpha and theta band with decreasing alpha responses in litFUS as compared to sham (comparing the top timefrequency plots within Figure 3 and Figure 2a).

\subsection{Hypothesis testing}

Table 1 summarizes the first subset of random intercept models. It shows that no increase in model fit was accomplished by introducing the depicted emotion of the stimulus into the model. The same is true for the difference between implicit vs. explicit processing of features. However, in general the significance of model 3 and 5 imply significant contribution of probabilistic learning and litFUS to the synchronization of alpha at the riFG.

Table 2 shows the full fixed effects estimates of model A5. The parameters are organized in ascending order of uncorrected $p$ values. After Bonferroni-Holm correction,

\begin{tabular}{rrlllrr} 
& Df & AIC & BIC & \multicolumn{1}{l}{$\chi^{2}$} & Df $\left(\chi^{2}\right)$ & $p\left(\chi^{2}\right)$ \\
A1 & 4 & 28513 & 28550 & & & \\
A2 & 5 & 28515 & 28559 & 0.3968 & 1 & .529 \\
A3 & 9 & 28475 & 28548 & 48.172 & 4 & $<.001^{* * *}$ \\
A4 & 15 & 28482 & 28599 & 4.827 & 6 & .566 \\
A5 & 27 & 28238 & 28442 & 267.853 & 12 & $<.001^{* * *}$
\end{tabular}

Table 1

Summary of model comparisons with alpha power density at F8 electrode position as dependent variable. $A 1=$ random intercept + random slope (trial), A2= $\mathrm{A} 1+$ emotion, $\mathrm{A} 3=\mathrm{A} 2+\operatorname{rate}(\text { angry })^{*}$ rate (male) + emotion* rate(angry), $\mathrm{A} 4=$ rate(angry)* rate(male)* block + emotion* rate(angry)* block, $\mathrm{A} 5=$ rate(angry)* rate (male)* Block*litFUS+ emotion* rate(angry)* block*litFUS; DF=degrees of freedom, AIC $=$ Akaike information criterion, $\mathrm{BIC}=$ Bayesian information criterion. $\operatorname{DF}\left(\chi^{2}\right)=$ degrees of freedom for the $\chi^{2}$ test. ${ }^{*}=$ $p<.05{ }^{* * *}=p<.001$

significant effects were in general found for the influence of feature rates, litFUS and block but not the current emotion depicted in a given trial. All main effects are estimated with respect to the reference categories of current emotion (neutral), block (attention on emotion) and litFUS (sham).

Just like the main effects, the interactions indicate the EEG alpha response at the riFG to change as a function of stochastic processing of stimulus features and attention focus but not processing of current emotional stimuli. Furthermore, litFUS introduces differences for all variables but the current emotion, again highlighting the riFG's role in stochastic processing.

In summary, Table 2 indicates higher rates of male and angry faces to decrease the alpha response. However, before the properties of the mixed model estimation procedure, these effects result in context of the reference categories of nominal predictors that are not included into the effect's (interaction-)term. Thus, higher rates of male and angry faces were found to decrease alpha reaction in sham sessions during trials with explicit focus on the depicted emotion. 
Processing of Emotional Features in the Lateral Frontal Cortex

$\begin{array}{llllllll} & \text { Estimate } & \text { SE } & \text { Df(Satt.) } & t(\text { Satt.) } & p \text { (Satt.) } & h o l m & \beta \\ \text { (Intercept) } & 16.160 & 0.863 & 5795.307 & 18.729 & <.001 & & -0.100 \\ \text { Rate(male) } & -12.436 & 1.740 & 8200.946 & -7.148 & <.001 & <.001 & -0.100 \\ \text { Rate(male):Block } & 12.548 & 1.772 & 8615.182 & 7.080 & <.001 & <.001 & 0.100 \\ \text { Block } & -6.032 & 0.872 & 8765.966 & -6.915 & <.001 & <.001 & 0.060 \\ \text { Rate(Angry) } & -11.730 & 1.733 & 8429.161 & -6.770 & <.001 & <.001 & -0.040 \\ \text { litFUS:Rate(male) } & 17.531 & 2.652 & 9766.579 & 6.611 & <.001 & <.001 & 0.220 \\ \text { Rate(Angry):Rate(male):Block } & -23.484 & 3.553 & 8335.600 & -6.610 & <.001 & <.001 & -0.100 \\ \text { Rate(Angry):Rate(male) } & 23.010 & 3.494 & 8038.097 & 6.585 & <.001 & <.001 & 0.100 \\ \text { litFUS:Rate(male):Block } & -17.276 & 2.693 & 9944.537 & -6.415 & <.001 & <.001 & -0.190 \\ \text { litFUS } & -8.357 & 1.306 & 9595.001 & -6.399 & <.001 & <.001 & 0.180 \\ \text { Rate(Angry):Block } & 11.450 & 1.792 & 8663.708 & 6.391 & <.001 & <.001 & 0.007 \\ \text { litFUS:Block } & 8.443 & 1.333 & 9841.110 & 6.334 & <.001 & <.001 & -0.060 \\ \text { Rate(Angry):litFUS:Rate(male):Block } & 30.286 & 5.767 & 9815.728 & 5.251 & <.001 & <.001 & 0.130 \\ \text { Rate(Angry):litFUS:Block } & -15.013 & 2.876 & 9729.021 & -5.220 & <.001 & <.001 & -0.020 \\ \text { Rate(Angry):litFUS:Rate(male) } & -29.499 & 5.709 & 9719.230 & -5.167 & <.001 & <.001 & -0.120 \\ \text { Rate(Angry):litFUS } & 14.542 & 2.826 & 9581.805 & 5.146 & <.001 & <.001 & 0.020 \\ \text { litFUS:Block:Emotion } & 0.444 & 0.465 & 10870.160 & 0.955 & 0.340 & 1 & 0.100 \\ \text { Rate(Angry):litFUS:Block:Emotion } & -0.687 & 0.999 & 10869.744 & -0.687 & 0.492 & 1 & -0.040 \\ \text { Block:Emotion } & -0.201 & 0.327 & 10868.876 & -0.614 & 0.539 & 1 & -0.070 \\ \text { Rate(Angry):Emotion } & -0.250 & 0.557 & 10870.987 & -0.450 & 0.653 & 1 & -0.010 \\ \text { Emotion } & 0.114 & 0.260 & 10870.395 & 0.437 & 0.662 & 1 & -0.001 \\ \text { Rate(Angry):litFUS:Emotion } & 0.312 & 0.831 & 10873.017 & 0.376 & 0.707 & 1 & 0.020 \\ \text { litFUS:Emotion } & -0.145 & 0.386 & 10872.477 & -0.375 & 0.708 & 1 & -0.001 \\ \text { Rate(Angry):Block:Emotion } & 0.248 & 0.701 & 10868.809 & 0.354 & 0.724 & 1 & 0.010\end{array}$

Table 2

Fixed effect estimates of model A5. Effects are presented in ascending order of their initial level of significance. A total of 10946 observations nested within 37 individuals was included in this analysis. Estimates reflect unstandardized effects, $\beta$ indicates standardized effects, SE=standard error, $\operatorname{Df}($ Satt $)=$ Satterwaite degrees of freedom, $\mathrm{p}$ (Satt.)= Satterwaite based $\mathrm{p}$ statistic. holm $=$ holm- adjusted $p$-value, $* * *$ indicates significance with $p<.001$,

In addition, litFUS produced greater differences between explicit and implicit processing of features. This effect was greater in trials targeting emotion processing. Finally, a complex four way interaction was found, indicating litFUS to alter the influence of a complex interplay of stimulus features (sex vs. emotion) for trials of explicit emotion processing. This interaction is illustrated in Figure 4.

Next, in order to clarify the role of alpha power in the processing of emotional faces, RTs were analyzed via a second set of random intercept models. The model comparison is summarized in Table 4.

$\begin{array}{rrrrrrr} & \text { Df } & \text { AIC } & \text { BIC } & \chi^{2} & D f\left(\chi^{2}\right) & p\left(\chi^{2}\right) \\ \text { B1 } & 5 & 14454 & 14490 & & & \\ \text { B2 } & 8 & 14272 & 14330 & 187.868 & 3 & <.001^{* * *} \\ \text { B3 } & 12 & 14270 & 14358 & 9.580 & 4 & .048^{*} \\ \text { B4 } & 20 & 14257 & 14403 & 29.244 & 8 & <.001^{* * *} \\ \text { B5 } & 22 & 14199 & 14360 & 61.685 & 2 & <.001^{* * *}\end{array}$

Table 3

Summary of model comparisons with reaction time as dependent variable. $\mathrm{DF}=$ degrees of freedom, $\mathrm{AIC}=$ Akaike information criterion, $\mathrm{BIC}=$ Bayesian information criterion. $\operatorname{DF}\left(\chi^{2}\right)=$ degrees of freedom for the $\chi^{2}$ test. ${ }^{*}=(p)<.05 .{ }^{* *}=(p)<.01$. $* * *=p<.001$. B1 =random intercept (for each participant) + random slope (trial). $\mathrm{B} 2=\mathrm{B} 1+$ change of emotion from previous to current trial). $\mathrm{B} 3=\mathrm{B} 2+$ Block. $\mathrm{B} 4=\mathrm{B} 3+$ litFUS. $\mathrm{B} 5=\mathrm{B} 4+\mathrm{Alpha}(\mathrm{F} 8)$
Since the best fit was given by model B5, it was further analyzed for fixed effects. Table 4 shows a significant difference between the angry $\rightarrow$ neutral sequence in comparison to neutral $\rightarrow$ neutral and neutral $\rightarrow$ angry. Furthermore, Alpha showed a highly significant contribution to improved performance. However, as alpha activity is negatively correlated to cortical activity, this implies inactivity of the right IPFC to decrease the reaction time, increasing processing speed.

\subsection{Additional analyses}

Since frontal alpha (FA) asymmetry measures have been shown to react to emotional stimuli, FA measures calculated from alpha at F7 and F8 electrode position were analyzed. Frontal alpha asymmetry was significantly predicted by depressivity and the individual alpha peak frequency as well as litFUS. The results of the calculation are shown in Table 5. They are illustrated in Figure 5. It shows a general increase of frontal alpha asymmetry from lower to higher IAF. Furthermore, during sham sessions, higher BDI-V measures correlate with lower frontal alpha asymmetry in those who have IAFs below $10 \mathrm{~Hz}$. This effect is reveresed by litFUS. Nonetheless, in individuals, who show higher IAF in litFUS sessions, higher BDI-V scores once again correlate to less positive FA measures even though the overall level of FA constantly rises from low to high asymmetry scores.

Furthermore, the same analysis from model A5 was performed again with theta at the F8 electrode position as dependent variable, since the time-frequency plots indicated 


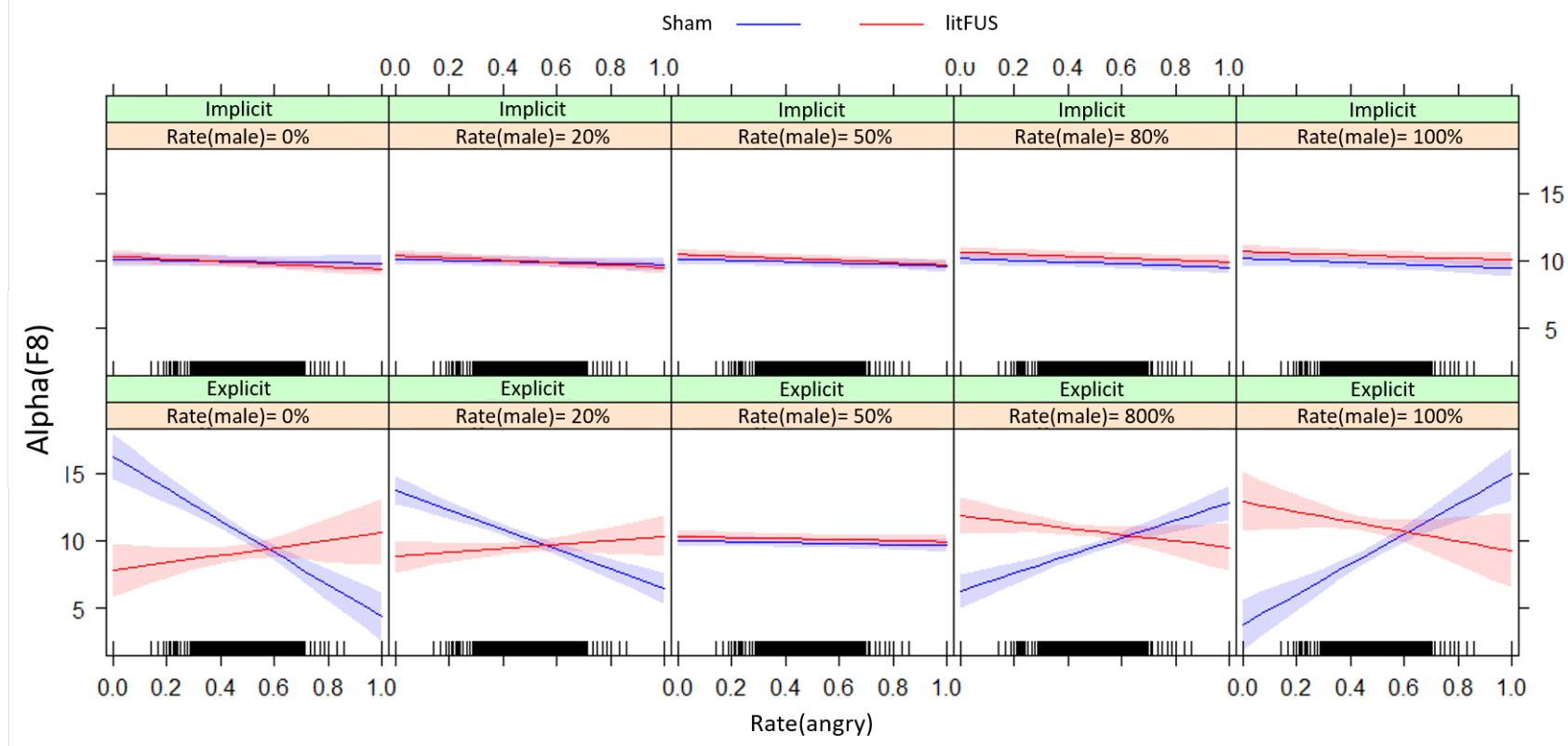

Figure 4: Estimated effects of the four-way interaction described in Table 3. In trials with focus on emotion processing (explicit), the alpha response to the stimulus in a given trial depends on the likelihood of previously presented male and angry faces while it does not in trials with a focus on sex (implicit [emotion processing]). The interaction of sex and emotion depiction rates inverses from low angry men rates to high angry men rates. This effect is upturned in litFUS compared to sham sessions.

$\begin{array}{llllllll} & \text { Estimate } & \mathrm{SE} & \mathrm{df} \text { (Satt.) } & \mathrm{t} & p & \text { holm } & \beta \\ (\text { Intercept }) & -0.023465 & 0.040596 & 39.142991 & -0.578 & 0.56656 & & -0.110 \\ \text { Alpha(F8) } & -0.043 & 0.006 & 10709.550 & -7.294 & <.001 & <.001 & -0.210 \\ \mathrm{~N} \rightarrow \mathrm{N}-\mathrm{A} \rightarrow \mathrm{N} & -0.113 & 0.024 & 10860.136 & -4.685 & <.001 & <.001 & -0.200 \\ \mathrm{~N} \rightarrow \mathrm{A}-\mathrm{A} \rightarrow \mathrm{N} & -0.105 & 0.027 & 10860.006 & -3.896 & <.001 & .001 & -0.200 \\ \text { litFUS } & 0.036 & 0.013 & 10897.968 & 2.854 & .004 & .060 & 0.070 \\ \text { Block } & -0.037 & 0.013 & 10876.731 & -2.757 & .006 & .076 & -0.070 \\ \text { Block:litFUS } & 0.033 & 0.018 & 10882.173 & 1.835 & .067 & .799 & 0.060 \\ \mathrm{~N} \rightarrow \mathrm{A}-\mathrm{A} \rightarrow \mathrm{N}: \text { Block:litFUS } & -0.078 & 0.052 & 10860.404 & -1.493 & .136 & 1 & -0.150 \\ \text { litFUS:Alpha(F8) } & 0.011 & 0.007 & 10895.188 & 1.419 & .156 & 1 & 0.030 \\ \mathrm{~A} \rightarrow \mathrm{A}-\mathrm{A} \rightarrow \mathrm{N}: \text { :lock } & -0.041 & 0.036 & 10855.413 & -1.125 & .260 & 1 & -0.080 \\ \mathrm{~N} \rightarrow \mathrm{A}-\mathrm{A} \rightarrow \mathrm{N} \text { :litFUS } & 0.033 & 0.036 & 10860.077 & 0.911 & .362 & 1 & 0.060 \\ \mathrm{~A} \rightarrow \mathrm{A}-\mathrm{A} \rightarrow \mathrm{N} & 0.021 & 0.025 & 10855.408 & 0.838 & .402 & 1 & 0.040 \\ \mathrm{~N} \rightarrow \mathrm{N}-\mathrm{A} \rightarrow \mathrm{N} \text { :Block } & -0.028 & 0.036 & 10859.545 & -0.800 & .424 & 1 & -0.050 \\ \mathrm{~N} \rightarrow \mathrm{N}-\mathrm{A} \rightarrow \mathrm{N}: \text { Block1:litFUS } & -0.023 & 0.048 & 10860.272 & -0.466 & .641 & 1 & -0.040 \\ \mathrm{~A} \rightarrow \mathrm{A}-\mathrm{A} \rightarrow \mathrm{N} \text { : Block:litFUS } & -0.021 & 0.049 & 10855.492 & -0.427 & .669 & 1 & -0.040 \\ \mathrm{~N} \rightarrow \mathrm{A}-\mathrm{A} \rightarrow \mathrm{N} \text { :Block } & 0.010 & 0.039 & 10859.084 & 0.257 & .797 & 1 & 0.020 \\ \rightarrow \mathrm{N}-\mathrm{A} \rightarrow \mathrm{N}: \text { litFUS } & -0.005 & 0.033 & 10858.838 & -0.157 & .875 & 1 & -0.010 \\ \mathrm{~A} \rightarrow \mathrm{A}-\mathrm{A} \rightarrow \mathrm{N} \text { :litFUS } & -0.004 & 0.034 & 10855.628 & -0.113 & .910 & 1 & -0.007\end{array}$

Table 4

Fixed effect estimates of B5 with RT as dependent variable. Effects are presented in ascending order of their initial level of significance. A total of 10946 trials nested within 37 test persons was used for this analysis. Estimates reflect unstandardized effects, $\beta$ indicates standardized effects, $\mathrm{SE}=$ standard error, Df (Satt)= Satterwaite degrees of freedom, $\mathrm{p}($ Satt.) $=$ Satterwaite based $\mathrm{p}$ statistic. $* *$ indicates significance with $\mathrm{p}<.01, * * *$ with $\mathrm{p}<.001$

that significant differences may also be found at these frequencies. The picture is very similar to the results in alpha. it is noticeable that the effect sizes are smaller. The effects are summarized in Table 6.

\section{Discussion}

The results depicted in section 3.1 show that the alpha response at F8 electrode position (indicating cortical inhibition of the riFG) is significantly modulated by the recent history of feature occurrence, regardless of it being a direct emotional stimulus or not (rate of angry faces vs. rate of depicted men). Nonetheless, the direction of this effect 
Processing of Emotional Features in the Lateral Frontal Cortex

$\begin{array}{llllll} & \text { Estimate } & \text { SE } & \text { Df } & \mathrm{t} & \mathrm{p} \\ \text { (Intercept) } & -2.410 & 0.919 & 1742.107 & -2.622 & 0.009 \\ \text { litFUS } & -6.963 & 0.505 & 10936.457 & -13.792 & <.001 \\ \text { IAF:litFUS } & 0.691 & 0.049 & 10936.169 & 14.136 & <.001 \\ \text { BDIV:IAF:litFUS } & -0.016 & 0.002 & 10923.581 & -7.204 & <.001 \\ \text { BDIV:litFUS } & 0.159 & 0.023 & 10923.218 & 7.058 & <.001 \\ \text { IAF } & 0.231 & 0.087 & 3355.062 & 2.664 & 0.008 \\ \text { BDIV } & -0.014 & 0.039 & 2342.107 & -0.351 & 0.726 \\ \text { BDIV:IAF } & 0.001 & 0.004 & 4206.840 & 0.149 & 0.882\end{array}$

Table 5

Fixed effects estimates with frontal alpha asymmetry as dependent variable. Effects are presented in ascending order of their level of significance. Estimates reflect unstandardized effects, $S E=$ standard error, Df (Satt)= Satterwaite degrees of freedom, $p$ $($ Satt. $)=$ Satterwaite based $\mathrm{p}$ statistic. ${ }^{* *}$ indicates significance with $\mathrm{p}<.01, * * *$ with $\mathrm{p}<.001$

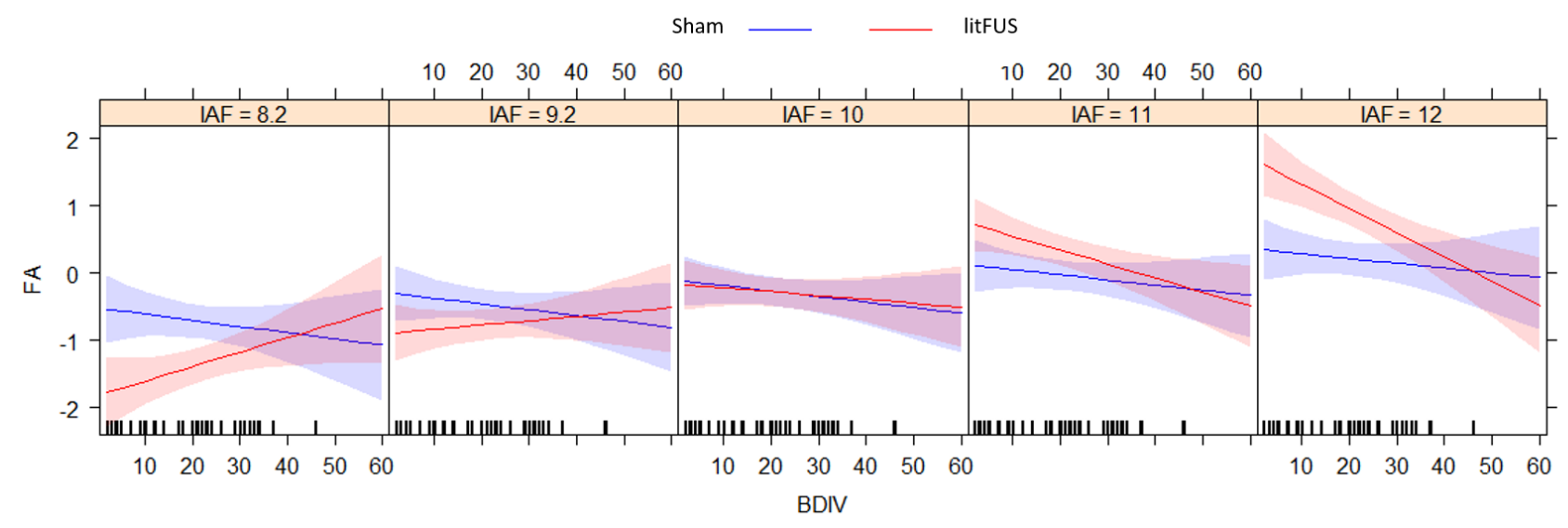

Figure 5: Frontal alpha asymmetry as predicted by litFUS, IAF and BDI-V scores. Frontal alpha asymmetry was calculated by subtracting $\ln ($ Alpha at F7) from $\ln ($ Alpha at F8). In sham sessions, higher depression scores were associated with less positive frontal asymmetry measures. In addition, higher individual alpha peak frequencies (IAF) predicted this effect to be diminished. litFUS reverses the connection between BDI-V and frontal asymmetry while increasing the estimated lower bound of effect lineally with the IAF.

seems to depend on several parameters including overall susceptibility of a neural response (manipulated by litFUS), an interaction of extracted features (task-relevant or irrelevant; sex vs. emotion) and focus of attention (manipulated by implicit vs. explicit processing blocks of trials for a given feature).

Accordingly, a predicted four-way interaction was found. Here not only the four-way but also all lower order interactions proved significant. On the other hand, no significant interaction was found for the set of interaction terms that included the currently depicted emotion. In addition all main effects with the exception of the currently depicted emotion also showed a highly significant influence. We therefore conclude that especially stochastic feature processing may take place in or be modulated by riFG neuronal ensembles, while processing of the currently depicted emotional value of a stimulus by itself has no impact on its EEG alpha response. Thus, the riFG may contribute to emotion processing in the way that it provides an estimate of the recent history of relevant and irrelevant information, thereby introducing a proper model of the "world" for further evaluation of situations. This notion is suggested by the strict division of effects containing the current emotion and those that don't in terms of significance in Table 4. As this also includes litFUS related effects, this data provides strong evidence that a manipulation of the riFG does not interact with this variable. Conversely, litFUS exhibited significant interactions with all other parameters, indicating their effect to depend on the neural context, that is excitability. Thus, modulation of the riFG addressability by other neural structures or networks that lead to either excitation or inhibition of the riFG may regulate the learning ability of this area.

Furthermore, given that the neuromodulation from litFUS in this study was hypothesized to decrease the general excitability of the neural tissue underlying F8 electrode position, the main effect showing greater alpha responses in sham compared to litFUS trials is somewhat surprising. However, consulting the estimates of Table 4 reveals a number of interaction terms, showing opposite directions. This indicates that the effect of neural activity suppression leading to an conversely directed main effect may mostly rely on opposed neuronal recruitment between conditions that adds up to a residual (main) net excitation effect (also see Figure 1).Furthermore, as stated above, main effects are 
Processing of Emotional Features in the Lateral Frontal Cortex

$\begin{array}{llllllll} & \text { Estimate } & \mathrm{SE} & \mathrm{Df}(\text { Satt.) } & t \text { (Satt.) } & p \text { (Satt.) } & \text { holm } & \beta \\ \text { (Intercept) } & 15.975 & 0.942 & 5959.535 & 16.962 & <.001 & & \\ \text { Rate(male):Block } & 11.882 & 1.940 & 8152.066 & 6.124 & <.001 & <.001 & 0.090 \\ \text { Rate(male) } & -11.631 & 1.904 & 7687.199 & -6.109 & <.001 & <.001 & -0.100 \\ \text { Block } & -5.686 & 0.955 & 8319.962 & -5.955 & <.001 & <.001 & 0.050 \\ \text { Rate(Angry) } & -10.809 & 1.896 & 7937.959 & -5.700 & <.001 & <.001 & -0.030 \\ \text { Rate(Angry):Rate(male):Block } & -21.983 & 3.888 & 7840.178 & -5.653 & <.001 & <.001 & -0.090 \\ \text { Rate(Angry):Rate(male) } & 21.289 & 3.824 & 7509.047 & 5.568 & <.001 & <.001 & 0.080 \\ \text { litFUS } & -7.872 & 1.430 & 9286.778 & -5.504 & <.001 & <.001 & 0.180 \\ \text { litFUS:Block } & 8.009 & 1.460 & 9580.280 & 5.486 & <.001 & <.001 & -0.060 \\ \text { litFUS:Rate(male) } & 15.887 & 2.904 & 9489.703 & 5.470 & <.001 & <.001 & 0.200 \\ \text { Rate(Angry):Block } & 10.669 & 1.961 & 8205.003 & 5.440 & <.001 & <.001 & 0.004 \\ \text { litFUS:Rate(male):Block } & -15.668 & 2.950 & 9702.318 & -5.312 & <.001 & <.001 & -0.170 \\ \text { Rate(Angry):litFUS:Block } & -14.308 & 3.150 & 9451.373 & -4.542 & <.001 & <.001 & -0.060 \\ \text { Rate(Angry):litFUS } & 13.627 & 3.094 & 9272.175 & 4.404 & <.001 & <.001 & 0.050 \\ \text { Rate(Angry):litFUS:Rate(male):Block } & 27.254 & 6.317 & 9552.578 & 4.315 & <.001 & <.001 & 0.110 \\ \text { Rate(Angry):litFUS:Rate(male) } & -26.197 & 6.253 & 9435.563 & -4.190 & <.001 & <.001 & -0.100 \\ \text { litFUS:Block:Emotion } & 0.200 & 0.510 & 10871.984 & 0.393 & .695 & 1 & 0.090 \\ \text { Block:Emotion } & -0.130 & 0.359 & 10870.811 & -0.362 & .717 & 1 & -0.060 \\ \text { Rate(Angry):litFUS:Emotion } & -0.278 & 0.911 & 10875.202 & -0.306 & .760 & 1 & -0.010 \\ \text { litFUS:Emotion } & 0.125 & 0.423 & 10874.633 & 0.295 & .768 & 1 & -0.003 \\ \text { Rate(Angry):litFUS:Block:Emotion } & -0.176 & 1.096 & 10871.559 & -0.160 & .873 & 1 & -0.009 \\ \text { Rate(Angry):Block:Emotion } & 0.085 & 0.769 & 10870.758 & 0.111 & .912 & 1 & 0.004 \\ \text { Emotion } & -0.008 & 0.285 & 10872.487 & -0.028 & .978 & 1 & -0.004 \\ \text { Rate(Angry):Emotion } & 0.006 & 0.610 & 10873.120 & 0.010 & .992 & 1 & 0.000\end{array}$

Table 6

Fixed effect estimates following model A5 with Theta at F8 as dependent variable. Effects are presented in ascending order of their initial level of significance. A total of 11164 trials nested within 37 individuals was included in this analysis. Estimates reflect unstandardized effects, $\beta$ indicates standardized effects, $\mathrm{SE}=$ standard error, $\mathrm{Df}(\mathrm{Satt})=$ Satterwaite degrees of freedom, $\mathrm{p}$ $($ Satt. $)=$ Satterwaite based $\mathrm{p}$ statistic. $($ holm $)=$ holm- adjusted $(\mathrm{p})$-value, $* * *$ indicates significance with $(\mathrm{p})<.001$

estimated before the context of explicit emotion- focus and average feature rates. Hence, this effect may be interpreted more like a simple effect. As a result, a random intercept model including only litFUS as a fixed factor and trial as random slope, thus estimating the effect regardless of all other predictor values, reveals a hypothesis congruent effect that points in the opposite direction as it does in Table 4 (Estimate (SE): 0.221(0.018), $t(10912.95839)=12.53, p<.001 * * *)$. In brief, litFUS was thus found to increase the alpha response generally. Nonetheless, process specific alterations of this effect may also emerge.

Moreover, Figure 2 further shows this effect of overall excitability (the litFUS effect) to also affect the processing of non-emotional features in a condition that demanded participants to focus on emotion, while no such effect is seen in trials that govern attention focus to sex (illustrated by the changes in sham slopes across the top row of graphs). Thus, in summary, both features (sex and emotion) seem to interact with each other and with litFUS mostly in situations that include a subject's attention to emotional features. This finding is well in line with above mentioned theories on the specialized role of the right IPFC in emotion processing. However, taken together with the reported null-finding regarding effects that comprise the currently depicted emotion, this result leads to the conclusion that the riFG has a specialized role in providing contextual information for other processes of emotion processing, which may take place in other subdivisions of the (right) 1PFC. In other words, the riFG seems to provide a stochastic model of task- or goalrelevant and irrelevant features specifically if an emotional cue is in focus of attention but leaves the processing of said cue to other areas and networks. As a result, the IPFC as a whole may be especially suited to integrate current experiences with those of the recent history. However, whether this integration also takes place in the right hemisphere or how this function is exactly operationalized is beyond the scope of the current analyses and needs further investigation.

Furthermore, litFUS transmitted inhibition resulted in inverted directions of the sex-emotion interaction effect during explicit emotion processing trials. Such interaction effects may stem from sex-specific interactions that may have occurred on a number of levels: First [1] report female faces showing no affection to be perceived somewhat less negative (e.g. more cooperative and honest, less angry) than men, making the same expression. Second, an interaction of the gender of participants and sex of the depicted social cues may be present in so far as that either the opposite or same gender/sex might introduce significant emotions in participants. A few studies reported female participants to be generally more sensitive to male emotional expressions as compared to females' ([24]; [92]). Furthermore, others reported a general advantage of women compared to men in facial emotion recognition ([85], [63]). In addition, an increased autonomous response was found in men reacting to angry 
male faces as compared to women, reacting to the same stimuli ([60]), which may be part a greater defensive response in men to male threatening stimuli ([49]).As a result, the non-emotional feature sex may vary in its effect depending on the sample composition. However, as the inversion of effects by litFUS is a within-subject effect, even though the participant's gender may contribute to sham-related effects significantly, the litFUS induced alteration would still reflect a general process of neural excitement/inhibition rather than a sub-population based artifact. Nonetheless, the direction of effects and the related litFUS- induced digression from these may thus change in other samples. Since only a few male subjects took part in this study, no analysis concerning interactions of the volunteer's gender and the task was conducted as results would be insufficiently powered. Becker et al. ([10]) further state a general difference in the perception of angry male vs. angry female faces. Thus, from this point of view, intra-session reversing of effects (changes in intercept and slope of the shamlitFUS regression from left to right in Figure 2) may be interpreted in the way that angry men in general elicit other responses as do angry women, resulting in opposed effects for trials that follow lessno angry men and those that follow manyjust angry men. Hence, taking these studies into account, it is unknown whether (in this task) sex is an unemotional feature. However, as mentioned before, litFUS interestingly reverses this possibly confounded effect.

Following this, to make concluding remarks regarding this and other effects it may also be beneficial to give an impression of what increases or decreases in alpha activity actually reflect within the given task. Thus, its connection to RT was calculated. On that note, the underlying presumption for all further interpretations is that a decrease in RT (increase in speed) reflects advantages in processing capabilities:

In this set of data, increases in alpha at F8 contributed to decreases in RT. Furthermore, in comparison to the shift from angry to neutral faces a general increase of RT was present for all combinations of sequences other than angry $\rightarrow$ angry. Thus, even though the sequence neutral $\rightarrow$ angry also contains a shift between depicted emotions, detaching from the angry face still took significantly longer independently from implicit vs. explicit processing or litFUS. Moreover, even though in a currently given trial the sequence neutral $\rightarrow$ neutral comprises the same depicted emotion as does angry $\rightarrow$ neutral, participants still reacted slower. Hence, the slowing process did not result from differences in reaction to the currently shown valence. However, as no significant difference was present to angry $\rightarrow$ angry, these data indicate an overall decrease in reaction speed following trials including angry faces. This effect was not interacting with litFUS or focus of attention. Thus, we conclude this effect not to result from litFUS induced changes in attention but from processes that are not measured directly, such as processing depth.

Further evidence from additional analyses finally show similar results for theta activity as compared to above mentioned alpha activity. In general, theta related findings are pointing into the same direction as did alpha-focused results.
However, effect sizes were greater for effects in alpha response.

Lastly, frontal alpha asymmetry, an indicator of affect and motivational tendencies was analyzed as an inhibitory neuromodulation at F8 electrode position should impact the relative activity of right compared to left hemispheric activity. The subsequent analysis showed a strong interaction between depression scores, IAF and litFUS. While frontal asymmetry seems to decrease with increasing depression averaged over all trials, litFUS can reverse this effect. The "normal" direction of this effect (in sham sessions) is consistent with the existing literature (e.g. [70]). Also, the leftto-right increase in frontal asymmetry with increased IAF seems consistent with current theories on the correlation of IAF with cognitive resources and thus possibly resilience (e.g. [46]; [14]; [3]; [31]; [68]. these results suggest that the effects of litfus may produce different effects depending on depressivity and IAF. It should be emphasized that litfus may have negative effects on frontal asymmetry, especially for individuals with particularly low IAF who are not showing sings of depression. On the other hand, these findings also show that in the current (healthy sample) relatively more depressed individuals may also benefit particularly strongly from neuromodulation, depending on their IAF.

However, by integrating this finding into aforementioned results, the role of inactivity of the riFG for reaction speed should be reconsidered. In fact, this exploratory finding may help interpreting contradicting results concerning the role of the right $\mathrm{PFC}$ in RT based experiments like the go/no-go task.

\subsection{Summary}

The experimental neuromodulation of the riFG was carried out via litFUS. It produced an overall decrease in alpha power within the task and further revealed a complex pattern of covariates to interact with its effect. For instance, litFUS effects were substantially altered by estimated likelihoods of feature emergence (emotional and non-emotional). In general, analyses of these interactions strongly suggest that the riFG of the right IPFC shows a special sensitivity to stochastic learning, that is a representation of the estimated likelihood of certain features of stimuli throughout the task. However, the impact of this probabilistic learning was most prominent in trials that demanded participants to focus on emotional cues. Interestingly, the alpha response of the riFG did not rely on the currently presented stimulus, nor did it rely on its interaction with stochastic estimations, indicating the integration of present stimulus features into the recent history of experiences to take place somewhere else.

Furthermore, even though, in this study, neuromodulation was carried out via litFUS, we do emphasize the idea that similar conditions may be subject to the interference of other neural networks signaling contextual factors such as mood-effects. Thus, congruence effects of mood and learning or attention may either partly rely on the subsequent alteration in stochastic learning capabilities of the right IPFC or this capability is by itself a result of these congruence 
effects. Nonetheless, this study provided evidence for the role of the right $1 \mathrm{PFC}$ in the correlated processing of the recent history of stimulus presentation. In addition, other features, which are not part of the current focus of attention were found to significantly interact with probabilistic representations of emotional faces. Also, this effect was almost completely reversed depending on the excitability of neuronal tissue, which was minpulated via litFUS.

In addition, this data set provides evidence for differential slowing of RTs following aversive/ threatening stimuli.

\subsection{Limitations}

This study used emotional faces as stimulus material. As the reported interactions indicate, this study was possibly unable to test the differential sensitivity of the right IPFC for emotional as compared to unemotional features, since these may be confounded. Furthermore, even though on a single trial level more than 10,000 data points were used for calculations including only within-subjects effects (with the exception of the random intercept), larger studies replicating the effects with more (diverse) participants are needed.

\subsection{Conclusions}

This study provides evidence for a specialized role of the riFG in stochastic learning and the representation of recent experiences. Furthermore, the present data indicate this property is expressed depending on the neural excitability that was successfully altered via litFUS neuromodulation. Finally, these effects had stronger implications ind situations with an explicit focus on emotional features. In brief, the riFG may contribute to a emotional processing by providing probabilistic information for further processing of emotional stimuli in nearby compartments of the right DLFPC or other networks.

\section{Acknowledgements}

We thank Myriam Metzulat and Adrian Dernbach for the conscientious and reliable data acquisition.

\section{References}

[1] Reginald B. Adams Jr et al. "Emotion in the neutral face: A mechanism for impression formation?" In: Cognition and Emotion 26.3 (2012), pp. 431-441. ISSN: 0269-9931.

[2] John J. B. Allen and Samantha J. Reznik. "Frontal EEG asymmetry as a promising marker of depression vulnerability: Summary and methodological considerations". In: Current opinion in psychology 4 (2015), pp. 93-97. ISSN: 2352-250X.

[3] Efthymios Angelakis et al. "EEG neurofeedback: a brief overview and an example of peak alpha frequency training for cognitive enhancement in the elderly". In: The clinical neuropsychologist 21.1 (2007), pp. 110-129. ISSN: 1385-4046.

[4] Shruti Baijal and Narayanan Srinivasan. "Emotional and hemispheric asymmetries in shifts of attention: An ERP study". In: Cognition and Emotion 25.2 (2011), pp. 280-294. ISSN: 0269-9931.

[5] Michela Balconi and Chiara Ferrari. "Emotional memory retrieval. rTMS stimulation on left DLPFC increases the positive memories". In: Brain imaging and behavior 6.3 (2012), pp. 454-461. ISSN: 1931-7557.
[6] Aron K. Barbey, Michael Koenigs, and Jordan Grafman. "Dorsolateral prefrontal contributions to human working memory". In: cortex 49.5 (2013), pp. 1195-1205. ISSN: 0010-9452.

[7] Douglas Bates et al. "Fitting linear mixed-effects models using lme4". In: arXiv preprint arXiv:1406.5823 (2014).

[8] O. M. Bazanova and D. Vernon. "Interpreting EEG alpha activity". In: Neuroscience \& Biobehavioral Reviews 44 (2014), pp. 94-110. ISSN: 0149-7634.

[9] O. M. Bazanova and D. Vernon. "Interpreting EEG alpha activity". In: Neuroscience \& Biobehavioral Reviews 44 (2014), pp. 94-110. ISSN: 0149-7634.

[10] D. Vaughn Becker et al. "The confounded nature of angry men and happy women". In: Journal of personality and social psychology 92.2 (2007), p. 179. ISSN: 1939-1315.

[11] Joan C. Borod et al. "Facial asymmetry while posing positive and negative emotions: Support for the right hemisphere hypothesis". In: Neuropsychologia 26.5 (1988), pp. 759-764. ISSN: 0028-3932.

[12] Maximilien Chaumon, Dorothy V. M. Bishop, and Niko A. Busch. "A practical guide to the selection of independent components of the electroencephalogram for artifact correction". In: Journal of neuroscience methods 250 (2015), pp. 47-63. ISSN: 0165-0270.

[13] Anastasia Christakou et al. "Disorder-specific functional abnormalities during sustained attention in youth with attention deficit hyperactivity disorder (ADHD) and with autism". In: Molecular psychiatry 18.2 (2013), pp. 236-244. ISSN: 1476-5578.

[14] C. Richard Clark et al. "Spontaneous alpha peak frequency predicts working memory performance across the age span". In: International Journal of Psychophysiology 53.1 (2004), pp. 1-9. ISSN: 0167-8760.

[15] Michael W. Cole and Walter Schneider. "The cognitive control network: integrated cortical regions with dissociable functions". In: Neuroimage 37.1 (2007), pp. 343-360. ISSN: 1053-8119.

[16] Andrew W. Corcoran et al. "Toward a reliable, automated method of individual alpha frequency (IAF) quantification". In: Psychophysiology 55.7 (2018), e13064. ISSN: 0048-5772.

[17] James Danckert et al. "Right hemisphere brain damage impairs strategy updating”. In: Cerebral Cortex 22.12 (2012), pp. 2745-2760. ISSN: 1460-2199.

[18] Richard J. Davidson. "What does the prefrontal cortex "do" in affect: perspectives on frontal EEG asymmetry research". In: Biological psychology 67.1-2 (2004), pp. 219-234. ISSN: 0301-0511.

[19] Richard J. Davidson, Carrie Ellen Schaffer, and Clifford Saron. "Effects of lateralized presentations of faces on self-reports of emotion and EEG asymmetry in depressed and non-depressed subjects". In: Psychophysiology 22.3 (1985), pp. 353-364. ISSN: 0048-5772.

[20] Arnaud Delorme and Scott Makeig. "EEGLAB: an open source toolbox for analysis of single-trial EEG dynamics including independent component analysis". In: Journal of neuroscience methods 134.1 (2004), pp. 9-21. ISSN: 0165-0270.

[21] U. L.F. Dimberg and Maria Petterson. "Facial reactions to happy and angry facial expressions: Evidence for right hemisphere dominance”. In: Psychophysiology 37.5 (2000), pp. 693-696. ISSN: 00485772.

[22] Matthew L. Dixon et al. "Emotion and the prefrontal cortex: an integrative review”. In: Psychological bulletin 143.10 (2017), p. 1033. ISSN: 1939-1455.

[23] Rebecca Elliott et al. "The neural basis of mood-congruent processing biases in depression". In: Archives of general psychiatry 59.7 (2002), pp. 597-604. ISSN: 0003-990X.

[24] Roland J. Erwin et al. "Facial emotion discrimination: I. Task construction and behavioral findings in normal subjects". In: Psychiatry research 42.3 (1992), pp. 231-240. ISSN: 0165-1781. 
[25] Negar Fani et al. "Attention bias toward threat is associated with exaggerated fear expression and impaired extinction in PTSD". In: Psychological medicine 42.3 (2012), pp. 533-543. ISSN: 1469-8978.

[26] Alex Filipowicz, Britt Anderson, and James Danckert. "Adapting to change: The role of the right hemisphere in mental model building and updating". In: Canadian Journal of Experimental Psychology/Revue canadienne de psychologie expérimentale 70.3 (2016), p. 201. ISSN: 1878-7290.

[27] Paul C. Fletcher et al. "Responses of human frontal cortex to surprising events are predicted by formal associative learning theory". In: Nature neuroscience 4.10 (2001), pp. 1043-1048. ISSN: 1546-1726.

[28] John Fox et al. "Effect displays for linear, generalized linear, and other models". In: R package version 3 (2016).

[29] Philip A. Gable, Lauren B. Neal, and A. Hunter Threadgill. "Regulatory behavior and frontal activity: Considering the role of revisedBIS in relative right frontal asymmetry". In: Psychophysiology 55.1 (2018), e12910. ISSN: 0048-5772.

[30] E. Goeleven, R. de Raedt, and L. Leyman. "Verschuere (2008)". In: The Karolinska directed emotional faces: a validation study. Cognit. Emot 22 (), pp. 1094-1118.

[31] Thomas H. Grandy et al. "Individual alpha peak frequency is related to latent factors of general cognitive abilities". In: Neuroimage 79 (2013), pp. 10-18. ISSN: 1053-8119.

[32] Dirk Hagemann et al. "Resting brain asymmetry and affective reactivity: Aggregated data support the right-hemisphere hypothesis". In: Journal of Individual Differences 26.3 (2005), pp. 139-154. ISSN: 1614-0001.

[33] Stuart Hameroff et al. "Transcranial ultrasound (TUS) effects on mental states: a pilot study". In: Brain stimulation 6.3 (2013), pp. 409-415. ISSN: 1935-861X.

[34] Eddie Harmon-Jones and Philip A. Gable. "On the role of asymmetric frontal cortical activity in approach and withdrawal motivation: An updated review of the evidence". In: Psychophysiology 55.1 (2018), e12879. ISSN: 0048-5772.

[35] Eddie Harmon-Jones and John J. B. Allen. "Anger and frontal brain activity: EEG asymmetry consistent with approach motivation despite negative affective valence". In: Journal of personality and social psychology 74.5 (1998), p. 1310. ISSN: 1939-1315.

[36] Johannes Hewig. "Intentionality in frontal asymmetry research". In: Psychophysiology 55.1 (2018), e12852. ISSN: 0048-5772.

[37] Johannes Hewig et al. "The relationship of cortical activity and personality in a reinforced go-nogo paradigm". In: Journal of Individual Differences 26.2 (2005), pp. 86-99. ISSN: 1614-0001.

[38] Clay B. Holroyd and Akina Umemoto. "The research domain criteria framework: the case for anterior cingulate cortex". In: Neuroscience \& Biobehavioral Reviews 71 (2016), pp. 418-443. ISSN: 0149-7634.

[39] Emmanuel Jesulola et al. "Frontal alpha asymmetry as a pathway to behavioural withdrawal in depression: Research findings and issues". In: Behavioural brain research 292 (2015), pp. 56-67. DOI: 10.1016/j.bbr. 2015.05.058.

[40] Jennifer Adrienne Johnson, Antonio P. Strafella, and Robert J. Zatorre. "The role of the dorsolateral prefrontal cortex in bimodal divided attention: two transcranial magnetic stimulation studies". In: Journal of cognitive neuroscience 19.6 (2007), pp. 907-920. ISSN: 0898-929X.

[41] Jürgen Kayser and Craig E. Tenke. "Principal components analysis of Laplacian waveforms as a generic method for identifying ERP generator patterns: I. Evaluation with auditory oddball tasks". In: Clinical neurophysiology 117.2 (2006), pp. 348-368. ISSN: 13882457. DOI: $10.1016 / j . c l i n p h .2005 .08 .034$.
[42] Jürgen Kayser and Craig E. Tenke. "Principal components analysis of Laplacian waveforms as a generic method for identifying ERP generator patterns: II. Adequacy of low-density estimates". In: Clinical neurophysiology 117.2 (2006), pp. 369-380. ISSN: 1388-2457. DOI: $10.1016 / \mathrm{j} . \mathrm{clinph} .2005 .08 .033$.

[43] Nicholas J. Kelley et al. "The relationship of approach/avoidance motivation and asymmetric frontal cortical activity: A review of studies manipulating frontal asymmetry". In: International Journal of Psychophysiology 119 (2017), pp. 19-30. ISSN: 0167-8760.

[44] Rebecca Kerestes et al. "Prefrontal cortical response to emotional faces in individuals with major depressive disorder in remission". In: Psychiatry Research: Neuroimaging 202.1 (2012), pp. 30-37. ISSN: 0925-4927.

[45] Philipp M. Keune et al. "Frontal brain asymmetry in adult attentiondeficit/hyperactivity disorder (ADHD): extending the motivational dysfunction hypothesis". In: Clinical neurophysiology 126.4 (2015), pp. 711-720. ISSN: 1388-2457.

[46] Wolfgang Klimesch. "EEG-alpha rhythms and memory processes". In: International Journal of Psychophysiology 26.1-3 (1997), pp. 319340. ISSN: 0167-8760.

[47] Kristin Koller-Schlaud et al. "EEG Frontal Asymmetry and Theta Power in Unipolar and Bipolar Depression". In: Journal of affective disorders (2020). ISSN: 0165-0327.

[48] Kristin Koller-Schlaud et al. "Test-Retest Reliability of Frontal and Parietal Alpha Asymmetry during Presentation of Emotional Face Stimuli in Healthy Subjects". In: Neuropsychobiology (2020), pp. 1-9. ISSN: 0302-282X.

[49] Mariska E. Kret and Beatrice de Gelder. "A review on sex differences in processing emotional signals". In: Neuropsychologia 50.7 (2012), pp. 1211-1221. ISSN: 0028-3932.

[50] Jan Kubanek et al. "Ultrasound modulates ion channel currents". In: Scientific reports 6.1 (2016), pp. 1-14. ISSN: 2045-2322.

[51] Micayla F. Lacey, Lauren B. Neal, and Philip A. Gable. "Effortful control of motivation, not withdrawal motivation, relates to greater right frontal asymmetry". In: International Journal of Psychophysiology 147 (2020), pp. 18-25. ISSN: 0167-8760.

[52] Wynn Legon et al. "A retrospective qualitative report of symptoms and safety from transcranial focused ultrasound for neuromodulation in humans". In: Scientific reports 10.1 (2020), pp. 1-10. ISSN: 20452322.

[53] Wynn Legon et al. "Neuromodulation with single-element transcranial focused ultrasound in human thalamus". In: Human brain mapping 39.5 (2018), pp. 1995-2006. ISSN: 1065-9471.

[54] Wynn Legon et al. "Transcranial focused ultrasound modulates the activity of primary somatosensory cortex in humans". In: Nature neuroscience 17.2 (2014), pp. 322-329. ISSN: 1546-1726.

[55] Wynn Legon et al. "Transcranial focused ultrasound neuromodulation of the human primary motor cortex". In: Scientific reports 8.1 (2018), pp. 1-14. ISSN: 2045-2322.

[56] Lemke Leyman et al. "Influence of high-frequency repetitive transcranial magnetic stimulation over the dorsolateral prefrontal cortex on the inhibition of emotional information in healthy volunteers". In: Psychological medicine 39.6 (2009), p. 1019. ISSN: 1469-8978.

[57] Yingjie Li et al. "Abnormal functional connectivity of EEG gamma band in patients with depression during emotional face processing". In: Clinical neurophysiology 126.11 (2015), pp. 2078-2089. ISSN: 1388-2457.

[58] Evi de Lissnyder et al. "Internal cognitive control in clinical depression: General but no emotion-specific impairments". In: Psychiatry research 199.2 (2012), pp. 124-130. ISSN: 0165-1781. 
[59] Daniel Lundqvist, Anders Flykt, and Arne Öhman. "The Karolinska directed emotional faces (KDEF)". In: CD ROM from Department of Clinical Neuroscience, Psychology section, Karolinska Institutet 91.630 (1998), p. 2.

[60] Evalynn J. Mazurski et al. "Conditioning with facial expressions of emotion: effects of CS sex and age". In: Psychophysiology 33.4 (1996), pp. 416-425. ISSN: 0048-5772.

[61] Michael B. Miller and Monica Valsangkar-Smyth. "Probability matching in the right hemisphere". In: Brain and cognition 57.2 (2005), pp. 165-167. ISSN: 0278-2626.

[62] Andrea Mognon et al. "ADJUST: An automatic EEG artifact detector based on the joint use of spatial and temporal features". In: Psychophysiology 48.2 (2011), pp. 229-240. ISSN: 0048-5772.

[63] Barbara Montagne et al. "Sex differences in the perception of affective facial expressions: Do men really lack emotional sensitivity?" In: Cognitive processing 6.2 (2005), pp. 136-141. ISSN: 1612-4782.

[64] Robin Nusslock, Keegan Walden, and Eddie Harmon-Jones. "Asymmetrical frontal cortical activity associated with differential risk for mood and anxiety disorder symptoms: An RDoC perspective". In: International Journal of Psychophysiology 98.2 (2015), pp. 249261. ISSN: 0167-8760.

[65] Michael Plaksin, Eitan Kimmel, and Shy Shoham. "Cell-typeselective effects of intramembrane cavitation as a unifying theoretical framework for ultrasonic neuromodulation". In: eneuro 3.3 (2016).

[66] Michael I. Posner and Yoav Cohen. "Components of visual orienting". In: Attention and performance X: Control of language processes 32 (1984), pp. 531-556.

[67] Rudi de Raedt et al. "Neurocognitive effects of HF-rTMS over the dorsolateral prefrontal cortex on the attentional processing of emotional information in healthy women: an event-related fMRI study". In: Biological psychology 85.3 (2010), pp. 487-495. ISSN: 0301-0511.

[68] Ian S. Ramsay et al. "Individual alpha peak frequency is slower in schizophrenia and related to deficits in visual perception and cognition”. In: Scientific reports 11.1 (2021), pp. 1-9. ISSN: 20452322 .

[69] Guangming Ran et al. "Attention modulates neural responses to unpredictable emotional faces in dorsolateral prefrontal cortex". In: Frontiers in human neuroscience 10 (2016), p. 332. ISSN: 16625161.

[70] Samantha J. Reznik and John J. B. Allen. "Frontal asymmetry as a mediator and moderator of emotion: An updated review". In: Psychophysiology 55.1 (2018), e12965. ISSN: 0048-5772.

[71] Samantha J. Reznik et al. "A double-blind pilot study of transcranial ultrasound (TUS) as a five-day intervention: TUS mitigates worry among depressed participants". In: Neurology, Psychiatry and Brain Research 37 (2020), pp. 60-66. ISSN: 0941-9500.

[72] Johannes Rodrigues et al. "EPOS: EEG Processing Open-Source Scripts". In: Frontiers in Neuroscience 15 (2021), p. 663. ISSN: 1662-453X.

[73] Johannes Rodrigues et al. "Mind the movement: Frontal asymmetry stands for behavioral motivation, bilateral frontal activation for behavior". In: Psychophysiology 55.1 (2018), e12908. ISSN: 00485772 .

[74] Matthew E. Roser et al. "Right hemisphere dominance in visual statistical learning". In: Journal of cognitive neuroscience 23.5 (2011), pp. 1088-1099. ISSN: 0898-929X.

[75] Alvaro Sanchez et al. "Effects of tDCS over the right DLPFC on attentional disengagement from positive and negative faces: an eyetracking study". In: Cognitive, Affective, \& Behavioral Neuroscience 16.6 (2016), pp. 1027-1038. ISSN: 1530-7026.
[76] Alvaro Sanchez-Lopez et al. "Neurocognitive mechanisms behind emotional attention: Inverse effects of anodal tDCS over the left and right DLPFC on gaze disengagement from emotional faces". In: Cognitive, Affective, \& Behavioral Neuroscience 18.3 (2018), pp. 485-494. ISSN: 1531-135X. DOI: 10.3758/s13415-018-0582-8.

[77] Joseph L. Sanguinetti et al. "Transcranial Focused Ultrasound to the Right Prefrontal Cortex Improves Mood and Alters Functional Connectivity in Humans". In: Frontiers in human neuroscience 14 (2020), p. 52. ISSN: 1662-5161.

[78] Manfred Schmitt et al. "Messgüte des vereinfachten Beck-DepressionsInventars (BDI-V)”. In: Diagnostica 49.4 (2003), pp. 147-156.

[79] Manfred Schmitt et al. "Normwerte für das vereinfachte BeckDepressions-Inventar (BDI-V) in der Allgemeinbevölkerung". In: Diagnostica 52.2 (2006), pp. 51-59.

[80] D. J.A. Smit et al. "The relation between frontal EEG asymmetry and the risk for anxiety and depression". In: Biological psychology 74.1 (2007), pp. 26-33. ISSN: 0301-0511.

[81] Stephen D. Smith and M. Barbara Bulman-Fleming. "An examination of the right-hemisphere hypothesis of the lateralization of emotion". In: Brain and cognition 57.2 (2005), pp. 210-213. ISSN: 0278-2626.

[82] Jennifer L. Stewart et al. "Resting frontal EEG asymmetry as an endophenotype for depression risk: Sex-specific patterns of frontal brain asymmetry". In: Journal of abnormal psychology 119.3 (2010), p. 502. ISSN: 1939-1846.

[83] Elisabeth Stöttinger et al. "Statistical and perceptual updating: correlated impairments in right brain injury". In: Experimental brain research 232.6 (2014), pp. 1971-1987. ISSN: 0014-4819.

[84] Barbara G. Tabachnick and Linda S. Fidell. Using Multivariate Statistics: Pearson. Sixth edition, New International Edition. Harlow: Pearson Education Limited, 2013. ISBN: 9781292021317.

[85] Julian Thayer and Bjørn Helge Johnsen. "Sex differences in judgement of facial affect: A multivariate analysis of recognition errors". In: Scandinavian journal of psychology 41.3 (2000), pp. 243-246. ISSN: 0036-5564.

[86] Ryan Thibodeau, Randall S. Jorgensen, and Sangmoon Kim. "Depression, anxiety, and resting frontal EEG asymmetry: a metaanalytic review". In: Journal of abnormal psychology 115.4 (2006), p. 715. ISSN: 1939-1846.

[87] William J. Tyler. "The mechanobiology of brain function". In: Nature Reviews Neuroscience 13.12 (2012), pp. 867-878. ISSN: 1471-0048.

[88] Nikita van der Vinne et al. "Frontal alpha asymmetry as a diagnostic marker in depression: Fact or fiction? A meta-analysis". In: Neuroimage: clinical 16 (2017), pp. 79-87. ISSN: 2213-1582.

[89] Jan Wacker et al. "Trait BIS predicts alpha asymmetry and P300 in a Go/No-Go task". In: European Journal of Personality: Published for the European Association of Personality Psychology 24.2 (2010), pp. 85-105. ISSN: 0890-2070.

[90] Catherine Wallez and Jacques Vauclair. "Right hemisphere dominance for emotion processing in baboons". In: Brain and cognition 75.2 (2011), pp. 164-169. ISSN: 0278-2626.

[91] Robert E. Wheeler, Richard J. Davidson, and Andrew J. Tomarken. "Frontal brain asymmetry and emotional reactivity: A biological substrate of affective style". In: Psychophysiology 30.1 (1993), pp. 82-89. ISSN: 0048-5772.

[92] Tanja S. H. Wingenbach, Chris Ashwin, and Mark Brosnan. "Sex differences in facial emotion recognition across varying expression intensity levels from videos". In: PLoS one 13.1 (2018), e0190634. ISSN: 1932-6203. 
[93] Irene Winkler, Stefan Haufe, and Michael Tangermann. "Automatic classification of artifactual ICA-components for artifact removal in EEG signals". In: Behavioral and brain functions 7.1 (2011), p. 30. ISSN: 1744-9081.

[94] George Wolford, Michael B. Miller, and Michael Gazzaniga. "The left hemisphere's role in hypothesis formation". In: Journal of Neuroscience 20.6 (2000), RC64-RC64. ISSN: 0270-6474.

[95] World Health Organization. ICD-10 : international statistical classification of diseases and related health problems : tenth revision. 2004.

[96] Miroslaw Wyczesany, Jan Kaiser, and Robert J. Barry. "Cortical lateralization patterns related to self-estimation of emotional state". In: (2009).

[97] Li-Chuan Yang, Ping Ren, and Yuan-Ye Ma. "Anodal transcranial direct-current stimulation over the right dorsolateral prefrontal cortex influences emotional face perception". In: Neuroscience Bulletin 34.5 (2018), pp. 842-848. ISSN: 1673-7067.

[98] Ling Zhang et al. "High-frequency repetitive transcranial magnetic stimulation of the left dorsolateral prefrontal cortex restores attention bias to negative information in methamphetamine addicts". In: Psychiatry research 265 (2018), pp. 151-160. ISSN: 0165-1781. 